\title{
Hyaluronic Acid and Platelet Rich Plasma in Hip Osteoarthritis: Work in Progress \\ Michele Abate*
}

Department of Medicine and Science of Aging, University G. D'Annunzio, Chieti-Pescara, Italy

\section{Introduction}

In the last decade Hyaluronic Acid (HA) and, more recently, Platelet Rich Plasma (PRP) have been used in the treatment of hip osteoarthritis (OA) [1]. Sound basic research supports their therapeutic potential.

\section{Hyaluronic Acid [HA]}

Produced by synoviocytes, fibroblasts and chondrocytes, is the major chemical component of synovial fluid. It is essential for the viscoelastic properties of the fluid because of high viscosity, and has a protective effect on articular cartilage and soft tissue surfaces of joints [1].

In $\mathrm{OA}$, the concentration and the molecular weight of HA are reduced, resulting in synovial fluid of lower elasticity and viscosity: diluitional effects, reduced hyaluronan synthesis and free radical degradation are the factors which contribute to the lower concentrations of HA. When the visco elasticity of synovial fluid is reduced, the transmission of mechanical force to cartilage may increase its susceptibility to mechanical damage [2].

Therefore, the restoration of the normal articular homoeostasis is the rationale for $\mathrm{HA}$ administration into $\mathrm{OA}$ joints. Besides viscosupplementation, HA is provided of other positive biological properties (e.g. biosupplementation), such as anti-inflammatory and anti-nociceptive effects, normalization of endogenous HA synthesis, and chondroprotection [3].

\section{Platelet rich plasma}

PRP is obtained increasing, by means of different methods of centrifugation, the amount of platelets above the normal blood values. The basic principle underlying the PRP therapeutic activity is to deliver a large pool of signaling proteins, such as growth factors and other cytokines (PDGF, TGF-1, VEGF, HGF, IL-8, CXCL12, chemokines, angiopoietin, and MMPs) to the local milieu driving the tissue regeneration mechanisms. These regulatory proteins must be capable of interfering with various healing mechanisms that is influencing the inflammatory response, inducing cell migration and proliferation, and modulating angiogenesis [4].

\section{HA and hip OA}

In the clinical setting, the efficacy of intra-articular HA has been shown by several studies, which have partially clarified the criteria of selection of the patients, and the schedule of administration of HA preparations [1].

In the majority of studies, patients with intermediate KellgrenLawrence (K-L) score were recruited, with the exclusion of subjects with evidence of rapidly destructive hip OA, absence of any articular space, and hypersensitivity to HA.

In these studies, both low and high molecular weight HA preparations were used; the numbers of injections ranged from 1 to 3 for each patients, with an interval between injections from 1 to 4 weeks. The number of injection needed is in general lower for high molecular weight HA preparation and this is not negligible advantage for the patients.
All the trials have shown pain reduction, which, in general, becomes evident within 3 months and persists in the following months. This is ascribed to the biological activities of HA, and is confirmed by the reduction of analgesics consumption. An early pain relief (within 1-3 weeks) is less frequent and is attributed to the viscoelastic properties of HA. Also the articular function is improved (better WOMAC and Lequesne Index, Walking Speed, Time to go up and down stairs).

Although at present all the characteristics of responders have not been clearly identified, some authors claim that a greater benefit may be obtained in patients with low and intermediate grade hip OA (K-L grade 2 and 3). On the contrary, age does not influence the therapeutic response [1].

Joint infiltration must be performed under strict ultrasound control, which allows a correct insertion of the needle in the capsular recess, and makes sure that HA is injected properly inside hip joint. Indeed, when HA is administered outside the articular space, treatment loses its efficacy and side effects, mainly pain, frequently occur [1].

\section{PRP and hip OA}

The clinical efficacy of PRP preparation in hip OA is supported by a limited number of clinical studies [5-8].

Sanchez et al. (2012) [8] report, in 40 patients, affected by monolateral severe hip OA, a statistically significant reduction in VAS, WOMAC and Harris hip subscores for pain and function after 7 weeks and 6 months. Side effects were negligible and were limited to a sensation of heaviness in the injection site.

These positive results have been confirmed by Civinini et al. (2013) [6] in a preliminary study, where 3 PRP infiltrations were performed and the follow-up lasted 12 months.

\section{HA/PRP versus other therapies}

Despite the positive results observed with HA and PRP infiltrations, it must be noticed that several studies are open observational studies, and only few studies have compared HA or PRP with placebo or other available treatments. A large trial, including 101 patients, comparing the efficacy of HA against corticosteroids and placebo, has shown only a limited efficacy of corticosteroids and HA in relieving pain in the short term, without any positive activity of both drugs in the long term [9]. Other studies have shown that the intra-articular injection of saline

*Corresponding author: Michele Abate, Department of Medicine and Science of Aging, University "G. d'Annunzio", Chieti-Pescara, Italy, Tel: +39 0871358 576; Fax: +39 0871358 969; E-mail: m.abate@unich.it

Received October 26, 2013; Accepted October 28, 2013; Published November 05, 2013

Citation: Abate M (2013) Hyaluronic Acid and Platelet Rich Plasma in Hip Osteoarthritis: Work in Progress. Surgery Curr Res 3: e110. doi:10.4172/21611076.1000e110

Copyright: (C) 2013 Abate M. This is an open-access article distributed under the terms of the Creative Commons Attribution License, which permits unrestricted use, distribution, and reproduction in any medium, provided the original author and source are credited. 
provides an important placebo effect (about $30 \%$ of pain reduction), which should be considered when estimating HA efficacy [10].

So, although it is well perceived that HA and PRP exert positive effects in the treatment of hip OA, up to now there is no strong evidence that HA is superior to other treatments, such as corticosteroids, physiotherapy or other conservative measures.

\section{PRP versus HA in hip OA}

Comparison trials between HA and PRP are also lacking. A single observation is present in the medical literature. This open study compared the clinical efficiency of autologous activated serum versus HA. Three intra-articular injections, once a week, of both agents were performed. After 3 months, the benefit was comparable, but, at 12 months, activated serum allowed a long-term preservation of achieved positive results [7].

\section{PRP versus HA in knee OA}

In contrast with hip OA, larger experience has been gained in the treatment of knee OA with biological agents. Indeed, the therapy with HA has been approved by the FDA, due to its superiority to placebo and other conservative treatments. Moreover, comparison studies between HA and PRP have been performed on knee OA [10].

Filardo et al. (2012) [11], in an open trial including 109 patients (55 treated with HA and 54 with PRP), at the follow-up evaluations (up to 12 months), observed in both groups a clinical improvement, but the comparison between the two groups showed a not statistically significant difference in all scores evaluated. A trend favorable for the PRP group was only found in patients with low grade articular degeneration (K-L score up to 2).

Similarly, in a prospective study including a total of 120 patients with K-L 1, 2, or 3 knee OA, found statistically significantly better results in the WOMAC and Numeric Rating Scale scores in the patients who received PRP injections after a 3 and 6 months follow-up, compared to those who were treated with HA [12].

Finally, Cerza et al. (2012) [13], performed a randomized controlled trial, where 60 patients received 4 intra-articular PRP injections, and 60 patients received 4 intra-articular HA injections, in a month period. Treatment with a local injection of PRP had a significant effect shortly after the final infiltration and a continuously improving sustained effect up to 24 weeks, as shown by WOMAC score. These results were better when compared with HA. In this latter group, the worst results were obtained for K-L 3 knee OA, whereas in the PRP patients no significant difference was observed in terms of the grade of OA.

Taken together, the results of comparative studies in knee OA, support the conclusion that in the long term PRP is superior to HA treatment.

On this basis, it should be very interesting to extend these observations to hip OA, which is as common and disabling condition as knee OA.

\section{Perspectives}

Recent experimental observations [8,14-18] suggest the possibility of a combined use of HA and PRP in the treatment of OA.

These studies have not specifically addressed this problem, but they strongly suggest a positive interaction between these biological agents.

Indeed, tendon and synovial fibroblasts migration, in vitro, is stimulated either by plasma rich in growth factors (PRGF) and HA, but the migratory ability is increased by the combination of these agents ( $+212 \%$ in comparison to PRGF alone, and $+335 \%$ compared with HA alone) [8].

In a rabbit model, cartilage fragments, loaded onto a scaffold composed of a HA derivative, human Fibrin Glue (FG) and autologous PRP, show an excellent proliferative capacity. At 6 months, in vivo, these cartilage fragment-loaded scaffolds provide an excellent cell source and induce significantly better repair tissue than the scaffold alone, as shown by histological scoring [16].

Finally, HA and PRP combined provide very good results in the healing of pressure ulcers $[8,14-18]$, and surgical wounds, but these anecdotal reports need confirmation on better structured studies.

\section{References}

1. Abate M, Pulcini D, Di lorio A, Schiavone C (2010) Viscosupplementation with intra-articular hyaluronic acid for treatment of osteoarthritis in the elderly. Curr Pharm Des 16: 631-640.

2. O’Regan M, Martini I, Crescenzi F, De Luca C, Lansing M (1994) Molecular mechanisms and genetics of hyaluronan biosynthesis. Int J Biol Macromol 16 283-286.

3. Takahashi K, Goomer RS, Harwood F, Kubo T, Hirasawa Y, et al. (1999) The effects of hyaluronan on matrix metalloproteinase-3 (MMP-3), interleukin1 beta(IL-1beta), and tissue inhibitor of metalloproteinase-1 (TIMP-1) gene expression during the development of osteoarthritis. Osteoarthritis Cartilage 7: 182-190.

4. Andia I, Abate M (2013) Platelet-rich plasma: underlying biology and clinical correlates. Regen Med 8: 645-658.

5. Battaglia M, Guaraldi F, Vannini F, Buscio T, Buda R, et al. (2011) Plateletrich plasma (PRP) intra-articular ultrasound-guided injections as a possible treatment for hip osteoarthritis: a pilot study. Clin Exp Rheumatol 29: 754.

6. Civinini R, Nistri L, Martini C, Redl B, Ristori G, et al. (2013) Growth factors in the treatment of early osteoarthritis. Clin Cases Miner Bone Metab 10: 26-29.

7. Noskov SM, Shirokova Llu, Snigireva AV, Parulia OM, Dybin SD (2012) [Efficiency of use of autologous activated serum in coxarthrosis]. Ter Arkh 84: 33-36.

8. Anitua E, Sanchez M, De la Fuente M, Zalduendo MM, Orive G (2012) Plasma rich in growth factors (PRGF-Endoret) stimulates tendon and synovial fibroblasts migration and improves the biological properties of hyaluronic acid. Knee Surg Sports Traumatol Arthrosc 20: 1657-1665

9. Colen S, van den Bekerom MP, Bellemans J, Mulier M (2010) Comparison of intra-articular injections of hyaluronic acid and corticosteroid in the treatment of osteoarthritis of the hip in comparison with intra-articular injections of bupivacaine. Design of a prospective, randomized, controlled study with blinding of the patients and outcome assessors. BMC Musculoskelet Disord 11: 264 .

10. Colen S, van den Bekerom MP, Mulier M, Haverkamp D (2012) Hyaluronic acid in the treatment of knee osteoarthritis: a systematic review and meta-analysis with emphasis on the efficacy of different products. BioDrugs 26: 257-268.

11. Filardo G, Kon E, Di Martino A, Di Matteo B, Merli ML, et al. (2012) Platelet-rich plasma vs hyaluronic acid to treat knee degenerative pathology: study design and preliminary results of a randomized controlled trial. BMC Musculoskelet Disord 13: 229.

12. Spaková T, Rosocha J, Lacko M, Harvanová D, Gharaibeh A (2012) Treatment of knee joint osteoarthritis with autologous platelet-rich plasma in comparison with hyaluronic acid. Am J Phys Med Rehabil 91: 411-417.

13. Cerza F, Carnì S, Carcangiu A, Di Vavo I, Schiavilla V, et al. (2012) Comparison between hyaluronic acid and platelet-rich plasma, intra-articular infiltration in the treatment of gonarthrosis. Am J Sports Med 40: 2822-2827.

14. de Angelis B, Lucarini L, Agovino A, Migner A, Orlandi F, et al. (2013) Combined use of super-oxidised solution with negative pressure for the treatment of pressure ulcers: case report. Int Wound J 10: 336-339.

15. De Angelis B, Lucarini L, Orlandi F, Agovino A, Migner A, et al. (2013) Regenerative surgery of the complications with Morton's neuroma surgery: use of platelet rich plasma and hyaluronic acid. Int Wound J 10: 372-376. 
Citation: Abate M (2013) Hyaluronic Acid and Platelet Rich Plasma in Hip Osteoarthritis: Work in Progress. Surgery Curr Res 3: e110. doi:10.4172/2161$1076.1000 \mathrm{e} 110$

16. Marmotti A, Bruzzone M, Bonasia DE, Castoldi F, Rossi R, et al. (2012) Onestep osteochondral repair with cartilage fragments in a composite scaffold. Knee Surg Sports Traumatol Arthrosc 20: 2590-2601.

17. Nicoli F, Balzani A, Lazzeri D, Gentile P, Chilgar RM, et al. (2013) Severe hidradenitis suppurativa treatment using platelet-rich plasma gel and Hyalomatrix. Int Wound J.
18. Yamada Y, Nakamura S, Ueda M, Ito K (2013) Papilla regeneration by injectable stem cell therapy with regenerative medicine: long-term clinical prognosis. $J$ Tissue Eng Regen Med. 